\title{
Mortality in hereditary antithrombin-III deficiency-1830 to 1989
}

\author{
F. R. ROSENDAAL H. HEIJBOER E. BRIËT H. R. BÚlleR \\ D. P. M. BRANDJES K. DE BRUIN D. W. Hommes
}

\section{J. P. VANDENBROUCKE}

To determine whether antithrombin-III (AT-III) deficiency leads to an excess mortality, we studied 171 individuals from ten families with a proven hereditary deficiency. 73 were classified as certainly deficient either by direct measurement of AT-III concentration or by mendelian inheritance patterns. 98 individuals had a high probability $(0.5)$ of deficiency. The 64 deaths recorded did not exceed those expected for the general population adjusted for age, sex, and calendar period. We suggest that a policy of prophylactic anticoagulation for patients with AT-III deficiency cannot be recommended.

Lancet 1991, 337: 260-62

\section{Introduction}

Hereditary antithrombin-III (AT-III) deficiency is an uncommon autosomal disorder that is associated with a tendency to venous thromboembolism in heterozygous individuals. ${ }^{12}$ The severity of venous thrombosis ranges from superficial thrombophlebitis to pulmonary embolism but the risk of severe thromboembolism in AT-III deficient individuals is largely unknown.

The decision to anticoagulate symptom-free AT-III deficient individuals prophylactically is therefore difficult and a randomised trial of anticoagulant treatment against placebo is unfeasible since the disorder is rare and a long

ADDRESSES Departments of Clinical Epidemiology ( $F \quad R$ Rosendaal, MD, $K$ de Bruin, BSc, J $P$ Vandenbroucke, $M D$ ) and Haematology (E Briẽt, MD, F R Rosendaal). University Hospital Leiden: and the Centre for Thrombosis. Haemostasis, and Atherosclerosis Research ( $H$ Heljboer, MD, H R Buller, MD, D P M Brandjes, MD, D W Hommes, BSc). Academic Medical Centre. Amsterdam, the Netherlands. Correspondence to DrF R Rosendaal, Department of Clinical Epıdemıology, Leiden University Hospital, Building 1 CO-P, PO Box 9600, 2300 RC Leiden, the Netherlands 
follow-up would be required to give a definitive answer. Furthermore, the risks of long-term anticoagulant treatment are substantral. ${ }^{3}$

To study the natural history of AT-III deficiency, we compared mortality in AT-III deficient families with that of the general population. If the mortality were lower in the general population, one could estumate the potentrally beneficial effect of anticoagulant treatment.

\section{Subjects and methods}

\section{Study populatıon}

All Dutch families, with a member known to be AT-III deficient and who was under the care of the Departments of Haematology of the Unversity Hospitals of either Leiden or Amsterdam, were eligible for study Diagnosis was based on AT-III antugen concentrations less than $75 \%$ of normal, in the absence of heparin treatment, chronic liver disease, or nephrotic syndrome Only families with 2 or more deficient individuals were studied, to avoid inclusion of patients with an acquired deficiency Furthermore, we included only tamiles with at least one symptomatc deficient individual

In all familes, non-deficient individuals were excluded and tor untested family members intormation from relatives was used to assign a probability of deficiency Since A'T-III deficiency is an autosomal disorder, some untested individuals can be taken as heterosygous for A'T-III deficiency-these family members have passed on the affected gene from common ancestors to deficient individuals. Mendelian probabulstes can be assigned to all individuals in a pedigree We restricted our study population to those who were deficient with certanty or had a probability of deficiency of 05 We rejected the possibility of recent mutations, which may theoretically have taken place in smaller pedigrees, because of the low frequency of AT-III deficiency. This method requires complete pedigrees. Since 1809 it has been mandatory by law in the Netherlands to report all births and deaths to the municipal registnes All information in this study has been venfied by reference to these muncipal and national registries

\section{Analysis}

The mortality of the study population (observed) was compared with the general population adjusted for age, sex, and calendar penoxd (expected) The ratio of observed to expected number of deaths $(\mathrm{OE})$ is the standardised mortality ratso (SMR) The expected mortality is derived by multuplying the total number of vears hived by the study population in each calendar period, and for each age and sex category, by the mortality rates (age and sex upecific) of the general population for each calendar period Confidence hmis for the SMR are based on a Porsson distribution tor the observed number of deaths ${ }^{4}$ 
Calendar periods were divided into twenty-year intervals from 1830 to 1989 , and to each of these we applied the population mortality rates of the mid-interval year, from 1840 onwards. In each calendar period we subdivided by sex and age class $(0-1$ yr, $1-4$ yrs, 5-9 yrs, and beyond in five-year age groups). Population mortality rates were obtained from the Central Bureau of Statistics. To eliminate bias that may have led to a spuriously low SMR, we ignored the first two decades of life, both for observed and expected mortality, the explanation being that those who passed on the gene to their descendents had to live until reproductive age before they could do so. This reason did not apply for those assigned a 0.5 probability of deficiency. In addition, we analysed mortality by age.

\section{Results}

We identified ten families with 2 or more AT-IIIdeficient individuals and at least one symptomatic deficient individual. The cutoff concentration for AT-III antigen of $75 \%$ of normal was satisfactory because all measurements were either less than $69 \%$ (taken as deficient) or greater than 83\% (normal). 73 classified as deficient with certainty: 23 based on information on family members (in 16 confirmed by AT-III measurement) and 50 based on low AT-III plasma concentrations. In addition, 98 individuals had a 0.5 probability of deficiency. No subject was lost to follow-up. The smallest family contributed 6 subjects to the study group, and the largest, 39 . Three to six generations were represented in these families. Birth year and year of death ranged from 1830 to 1989 and 1899 to 1987 , respectively.

Of a total of 8168 person-years (all ages combined), 64 deaths took place. There were 5066 person-years in the over 20 age group and 56 deaths. $65 \%$ of those who died were born before 1900 , while $55 \%$ died before 1950 . Mean age of death was 57 years, median $66 \mathrm{yr}$ ). The mortality in the study group did not differ from that of the general population. The expected number of deaths was 58.8 (aged 20 years and older $)$. The relative mortality was $1.0(\mathrm{O} / \mathrm{E}$, $56 / 58.8 ;$ SMR, $1 \cdot 0 ; 95 \%$ confidence interval, $0 \cdot 7-1 \cdot 2$ ). In the group classified as deficient with certainty there was no excess mortality: 9 deaths took place compared with 9.8 deaths expected (SMR, 0.9; 95\% CI, 0.4-1.6). Subjects with a 0.5 probability of deficiency also had a mortality frequency comparable with the general population $(\mathrm{O} / \mathrm{E}$, $47 / 48 \cdot 9 ; \mathrm{SMR}, 1 \cdot 0 ; 95 \% \mathrm{CI}, 0 \cdot 7-1 \cdot 3)$. For men $>20 \mathrm{yrs}$, the SMR was $0.9(\mathrm{O} / \mathrm{E}, 36 / 41.9 ; 95 \% \mathrm{CI}, 0.6-1 \cdot 2)$, and in women the SMR was $1 \cdot 2(\mathrm{O} / \mathrm{E}, 20 / 16.9 ; 95 \% \mathrm{CI}, 0.7-1.8)$. In addition, although a deficiency of AT-III may increase risk of postpartum thrombosis, there was no excess 


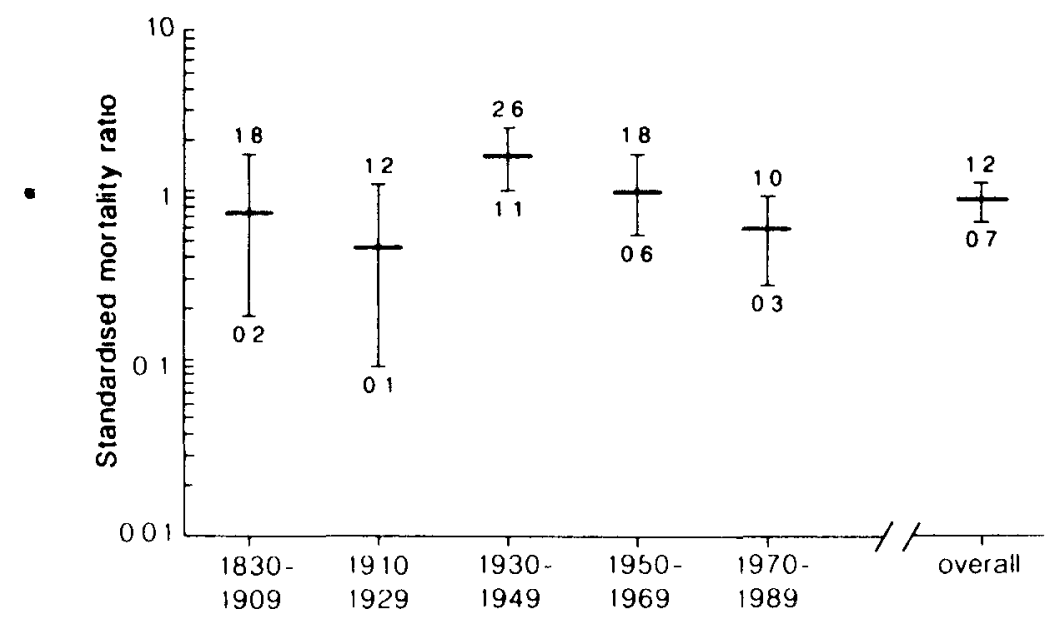

Fig 1-Mortality in AT-III deficiency by calendar period

mortality in women aged $20-40$ yrs $(\mathrm{O} / \mathrm{E}, 3 / 2.5 ; \mathrm{SMR}, 1 \cdot 2$; $95 \% \mathrm{CI}, 0 \cdot 2-3 \cdot 0)$.

Figure 1 shows the relative mortality for different calendar periods from 1830 to the present. Although some variation exists, AT-III deficiency does not appear to lead to excess mortality. Figure 2 shows the mortality ratio for different age groups and includes those subjects aged $\leqslant 19$. In this young group, relative mortality is low $(\mathrm{O} / \mathrm{E}, 8 / 27.5$; SMR, 0.3). This may be because of preferential inclusion of those who had children or under-reporting of child deaths in the nineteenth century. In all other age groups observed mortality was equal to expected mortality.

\section{Discussion}

We studied the natural history and mortality of AT-III deficiency since most individuals in our study group lived before anticoagulant therapy became available. No excess mortality was found and confidence intervals were narrow. In any genetic study there is a risk of bias and although the frequency of AT-III deficiency in the general population is low, the recruitment of affected family members was probably incomplete because not every individual will manifest venous thromboembolism. Incomplete data collection will lead to a biased estimate only if AT-IIP deficiency runs a different course in different families and breeds true within those families. Severely affected families are then more likely to be included than mildly affected families and so mortality may be overestimated. Since no excess mortality was found, this possibility is unlikely. Part of the study group only had a probability of being deficient 


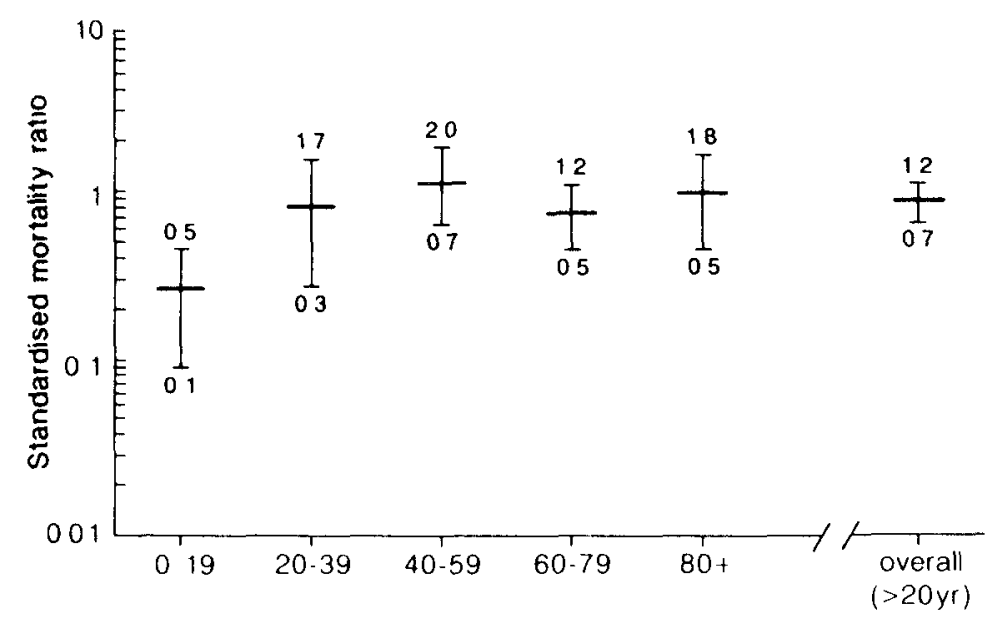

Fig 2-Mortality in AT-III deficiency by age

in AT-III and therefore some individuals may, in reality, have had normal AT-III concentrations. However, we found no excess mortality in the subgroup of individuals proven to be deficient. The inclusion of individuals with a 0.5 probability of deficiency allowed us to both increase the statistical power of the analysis by extending the study back to 1830 and reduce bias by selecting those who reproduced or were tested.

Life-long anticoagulant treatment is probably indicated after a first episode of thromboembolism. ${ }^{5.6}$ The need for long-term prophylaxis with anticoagulants in symptom-free AT-III deficient individuals is uncertain. ${ }^{7}$ Some groups recommend life-long prophylaxis in all deficient individuals, ${ }^{5}$ while others argue that anticoagulation should be reserved for those with additional risk factors for or a previous history of thromboembolism. ${ }^{6-8}$ Until now, evidence has been confined to case reports. Our study, in complete family pedigrees, suggests that life-long treatment with anticoagulants in symptom-free AT-III deficient individuals is unlikely to improve survival.

We thank members of all famlies in this study who kindly provided us with information and Mrs W. M. P. Noteboom for her diligence and perseverance in the genealogical research.

\section{REFERENCES}

1. Egeberg $O$. Inherited antithrombin deficiency causing thrombophilia. Thromb Diathes Haemor 1965; 13: 516-30.

2. Thaler E, Lechner $K$. Antithrombin III deficiency and thromboembolism. Clin Haematol 1981; 10: 369-91. 

3. Levine $M N$, Raskob G, Hirsh J. Hemorrhagic complications of long-term anticoagulant treatment. Chest 1989; 95 (Suppl): $26 S-36 S$.

4. Vandenbroucke JP. A shortcut method for calculating the 95 per cent confidence interval of the standardized mortality ratio. $A m \mathfrak{f}$ Epidemiol 1982; 115: 303-04.

5. Cosgriff TM, Bishop DT, Hershgold EJ, et al. Familial antithrombin III deficiency: its natural history, genetics, diagnosis and treatment. Medicine 1983; 62: 209-19.

6. Halal F, Quenneville G, Laurin S, Loulou G. Clinical and genetic aspects of antithrombin III deficiency. Am 7 Med Genet 1983; 14: 737-50.

7. Anonymous. Familial antithrombin-III deficiency. Lancet 1983; i: 1021-22.

8. Michiels JJ, Van Vliet HHDM. Hereditary antithrombin III deficiency and venous thrombosis. Neth $\mathcal{F}$ Med 1984; 27: 226-32. 